\title{
Dynamic Performance Characteristics of a Curved Slider Bearing Operating with Ferrofluids
}

\author{
Udaya P. Singh ${ }^{1}$ and R. S. Gupta ${ }^{2}$ \\ ${ }^{1}$ Department of Applied Science \& Humanities, Ambalika Institute of Management \& Technology, Lucknow 227305, India \\ ${ }^{2}$ Department of Mathematics, Kamala Nehru Institute of Technology, Sultanpur 228118, India
}

Correspondence should be addressed to Udaya P. Singh, journals4phd@gmail.com

Received 18 May 2012; Revised 15 July 2012; Accepted 22 July 2012

Academic Editor: Michel Fillon

Copyright ( 2012 U. P. Singh and R. S. Gupta. This is an open access article distributed under the Creative Commons Attribution License, which permits unrestricted use, distribution, and reproduction in any medium, provided the original work is properly cited.

\begin{abstract}
In the present theoretical investigation, the effect of ferrofluid on the dynamic characteristics of curved slider bearings is presented using Shliomis model which accounts for the rotation of magnetic particles, their magnetic moments, and the volume concentration in the fluid. The modified Reynolds equation for the dynamic state of the bearing is obtained. The results of dynamic stiffness and damping characteristics are presented. It is observed that the effect of rotation of magnetic particles improves the stiffness and damping capacities of the bearings.
\end{abstract}

\section{Introduction}

In the field of engineering and technology, slider bearings are often designed to bear the transverse loads. The study of performance characteristics of slider bearings with different shape and different lubricants has been done from time to time by the researchers. Gupta and Kavita [1] analysed the effect of frame rotation for porous slider bearing, Singh and Gupta [2] investigated the performance of a pivoted curved slider bearings for pseudoplastic and dilatant lubricants, Pascovici et al. [3] presented an experimental evidence of cavitation effects in a Rayleigh step slider, Venkateswarlu and Rodkiewicz [4] discussed the thrust bearing characteristics considering the terminal speed of the slider, Williams and Symmons [5] analysed the performance of hydrodynamic slider bearings for non-Newtonian lubricants, and Sharma and Pandey [6] presented an experimental comparison of the slider bearing performance for different shapes.

In last few decades, the researches shown that the performances of the bearings can be improved, and enhanced pressure and load carrying capacity can be obtained by use of the magnetic lubricants and magnetic fields [7-10]. The applications of magnetic lubricant are widely found in dampers, seals, sensors, loudspeakers, gauges, steppers, and coating systems [11]. Investigators have used the Jenkins model [12] for the lubricant flow. On the other hand, Shliomis $[13,14]$ proposed a ferrofluid flow model, in which the effects of rotation of magnetic particles, their magnetic moments, and the volume concentration are included. Ram and Verma [15] used the Shliomis model to investigate the performance of a porous inclined slider bearing and reported an increased pressure and load capacity. Shah and Bhat [16] used this model to study the ferrofluid-based squeeze film characteristics of curved annular plates and obtained similar results. Yamaguchi [17] presented a detailed analysis and simplification of the Shliomis model for different lubricating conditions. Recently, the Shliomis model was used by Lin [18] to study the squeeze film characteristics of circular plates under magnetic field. It was observed that the volume concentration and the intensity of magnetic field provide an increase in the load capacity and the time of approach.

All these researchers have analyzed the steady-state characteristics of the bearings lubricated with magnetic fluids. However, the study of the dynamic (damping and stiffness) characteristics of the bearings lubricated with magnetic fluids is yet to be investigated for its importance in the bearing design. 


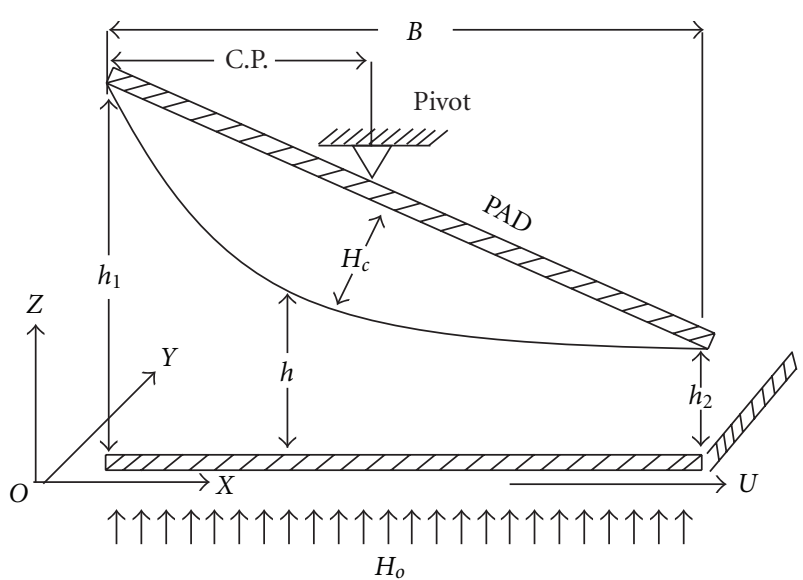

FIGURE 1: Schematic diagram of a curved slider bearing under applied magnetic field with a pivot at the centre of pressure (C.P.) and $H_{c}$ at $B / 2$.

In the present theoretical analysis, an attempt has been made to investigate the effects of the particle rotation and volume concentration of magnetic particles on the dynamic characteristics of curved slider bearings lubricated with magnetic fluids in the presence of transversely uniform magnetic fields.

\section{Constitutive Equations and Boundary Conditions}

The physical configuration of a curved slider bearing lubricated with a magnetic fluid in the presence of a transverse magnetic field is described in Figure 1. It is assumed that the thin film lubrication theory is applicable for the present analysis.

After the ferrohydrodynamic flow model by Shliomis [13, 14], the electromagnetic field equations are

$$
\begin{gathered}
\nabla \times \vec{H}=0, \\
\nabla \cdot(\vec{H}+\vec{M})=0,
\end{gathered}
$$

where $\vec{H}$ is the applied magnetic field and $\vec{M}$ is the magnetization vector.

Constitutive equations for incompressible magnetic fluid with internal rotation of magnetic particles $[13,14,17]$ are

$$
\begin{gathered}
\nabla \cdot \vec{v}=0, \\
\rho \frac{D \vec{v}}{D t}=-\nabla p+\eta \nabla^{2} \vec{v}+\mu_{o}(\vec{M} \cdot \nabla) \vec{H}+\frac{1}{2 \tau_{s}} \nabla \times(\vec{S}-I \vec{\Omega}),
\end{gathered}
$$

where $\vec{v}$ is the fluid velocity vector, $\rho$ is the fluid density, $t$ is the time, $p$ is the pressure, $\eta$ is the viscosity of the suspension, $\mu_{o}$ is the free space permeability, $\tau_{s}$ is the spin relaxation time, and $I$ is the sum of moments of inertia of the particles per unit volume. The local angular velocity of the $\vec{\Omega}$, equilibrium magnetization $\vec{M}$, and the internal angular moment $\vec{S}$ are given as

$$
\begin{gathered}
\vec{\Omega}=\frac{1}{2} \nabla \times \vec{v}, \\
\vec{M}=M_{o} \frac{\vec{H}}{H_{o}}+\frac{\tau_{B}}{I}(\vec{S} \times \vec{M}), \\
\vec{S}=I \vec{\Omega}+\mu_{o} \tau_{s}(\vec{M} \times \vec{H}) .
\end{gathered}
$$

where $\tau_{B}$ is the Brownian relaxation time of magnetic particles.

Assuming the slider to be infinitely wide, (2) for one dimensional flow under isothermal condition $[15,16]$ are

$$
\begin{gathered}
\frac{\partial u}{\partial x}+\frac{\partial w}{\partial z}=0, \\
\frac{\partial^{2} u}{\partial z^{2}}=\frac{1}{\eta\left(1+\mu_{o} M_{o} H_{o} G \bar{\tau} / 4 \eta\right)} \frac{\partial p}{\partial x}
\end{gathered}
$$

where

$$
\bar{\tau}=\frac{\tau_{B}}{1+\mu_{o} \tau_{B} \tau_{s} M_{o} H_{o} / I} .
$$

In the Langevin relationship, the magnetization depends upon the intensity of magnetic fields for a system of spherical particles $[13,14]$ and it is given as

$$
\begin{gathered}
M_{o}=n m\left(\operatorname{Coth}(\alpha)-\frac{1}{\alpha}\right), \\
\alpha=\frac{\mu_{o} m H_{o}}{k_{B} T}, \\
\tau_{s}=\frac{6 \eta \phi}{I}, \\
\tau_{B}=\frac{3 \eta \phi}{n k_{B} T},
\end{gathered}
$$

where $n$ is the number of magnetic particles per unit volume, $m$ is the magnetic moment of a particle, $\alpha$ is the Langevin parameter, $k_{B}$ is the Boltzmann constant, $T$ is the temperature, and $\phi$ is the volume fraction of the dispersed solid phase.

Substituting (6) and (7) in (5) and introducing the magnetoviscosity, the additional viscosity $\left(\eta_{m}\right)$ due to rotation of magnetic particles $[13,14]$ :

$$
\eta_{m}=\frac{3}{2} \eta \phi \frac{\alpha-\tanh (\alpha)}{\alpha+\tanh (\alpha)}
$$

(5) can be rewritten as

$$
\frac{\partial^{2} u}{\partial z^{2}}=\frac{1}{\eta+\eta_{m}} \frac{\partial p}{\partial x} .
$$


Integrating (9) under the no-slip boundary conditions:

$$
\begin{array}{ll}
u=U & \text { at } z=0, \\
u=0 & \text { at } z=h,
\end{array}
$$

the expression of $u$ is obtained as

$$
u=\frac{1}{\eta+\eta_{m}} \frac{\partial p}{\partial x}\left(z^{2}-z h\right)+U\left(1-\frac{z}{h}\right) .
$$

Substituting (11) in (4) and integrating under the boundary conditions:

$$
\begin{gathered}
w=0 \quad \text { at } z=0, \\
w=V=-\frac{\partial h}{\partial t} \quad \text { at } z=h,
\end{gathered}
$$

the modified Reynolds equation is obtained as

$$
\frac{\partial}{\partial x}\left(h^{3} \frac{\partial p}{\partial x}\right)=6\left(\eta+\eta_{m}\right) U \frac{\partial h}{\partial x}+12\left(\eta+\eta_{m}\right) \frac{\partial h}{\partial t} .
$$

Here, the effective viscosity $\eta[14]$ is given by

$$
\eta=\eta_{o}\left(1+\frac{4 \alpha+\tanh (\alpha)}{\alpha+\tanh (\alpha)} \phi\right)
$$

where $\eta_{0}$ denotes the viscosity of the carrier fluid.

For the one-dimensional curved slider bearings (Figure 1), the film thickness is taken as suggested by Abramovitz [19] in the form:

$$
h=H_{c}\left[4\left(\frac{x}{B}-\frac{1}{2}\right)^{2}-1\right]+\left[\left(h_{1}-h_{2}\right)\left(1-\frac{x}{B}\right)\right]+h_{2},
$$

which can be rewritten as

$$
h(x, t)=h_{s}(x)+h_{m}(t),
$$

where $h_{s}$ determines the steady state film profile, given by

$$
h_{s}=\frac{\left(h_{1}-h_{2}+4 H_{c}\right)^{2}}{16 H_{c}}-\frac{\left(h_{1}-h_{2}+4 H_{c}\right)}{B} x+\frac{4 H_{c}}{B^{2}} x^{2},
$$

and $h_{m}(t)$ is the time dependent minimum film thickness which, in steady state, can be given as

$$
h_{m}(0)=\left[1-\frac{(1-r)}{2}-\frac{(1-r)^{2}}{16 \delta}-\delta\right] h_{2},
$$

where, $\delta=H_{c} / h_{2}$ and $r=h_{1} / h_{2}$.

Introducing the dimensionless quantities:

$$
\begin{gathered}
\bar{x}=\frac{x}{B}, \quad \bar{h}=\frac{h}{h_{2}}, \quad \bar{h}_{m}=\frac{h_{m}}{h_{2}}, \\
\bar{h}_{s}=\frac{h_{s}}{h_{2}}, \quad \bar{p}=\frac{p h_{2}^{2}}{\eta_{o} U B}, \\
\bar{t}=\frac{U t}{B}, \quad \bar{V}=\frac{B V}{h U}=\frac{d \bar{h}_{m}}{d \bar{t}}, \\
\bar{\eta}=\frac{\eta}{\eta_{o}}, \quad \bar{\eta}_{m}=\frac{\eta_{m}}{\eta_{o}},
\end{gathered}
$$

the modified Reynolds equation can be expressed in dimensionless form:

$$
\begin{gathered}
\frac{\partial}{\partial \bar{x}}\left(\bar{h}^{3} \frac{\partial \bar{p}}{\partial \bar{x}}\right)=-6\left(\bar{\eta}+\bar{\eta}_{m}\right)[\{(1-4 \delta-r)+8 \delta \bar{x}\}+2 \bar{V}], \\
\bar{h}=\bar{h}_{S}+\bar{h}_{m}=\frac{(r-1+4 \delta)}{16 \delta}-(r-1+4 \delta) \bar{x}+4 \delta \bar{x}^{2}+\bar{h}_{m}(t) .
\end{gathered}
$$

Integrating (20) under the pressure boundary conditions $\bar{p}=$ 0 at $\bar{x}=0,1$, the film pressure is obtained as

$$
\begin{aligned}
\bar{p}=-6\left(\bar{\eta}+\bar{\eta}_{m}\right)[ & (1-4 \delta-r+2 \bar{V}) f_{1}(\bar{x}) \\
& \left.+4 \delta f_{2}(\bar{x})\right]+C f_{3}(\bar{x}),
\end{aligned}
$$

where

$$
\begin{gathered}
f_{1}(\bar{x})=\int_{0}^{\bar{x}} \frac{\bar{x}}{\bar{h}^{3}} d \bar{x}, \\
f_{2}(\bar{x})=\int_{0}^{\bar{x}} \frac{\bar{x}^{2}}{\bar{h}^{3}} d \bar{x}, \\
f_{3}(\bar{x})=\int_{0}^{\bar{x}} \frac{1}{\bar{h}^{3}} d \bar{x}, \\
C=6\left(\eta+\eta_{m}\right) \frac{(1-4 \delta-r+2 \bar{V}) f_{1}(1)+4 \delta f_{2}(1)}{f_{3}(1)} .
\end{gathered}
$$

The film force is obtained by integrating the film pressure over the slider length as follows:

$$
F=L \int_{x=0}^{B} p d x
$$

It takes the dimensionless form:

$$
\begin{aligned}
\bar{F}=\int_{0}^{1} \bar{p} d \bar{x} & \\
=-6\left(\bar{\eta}+\bar{\eta}_{m}\right)[ & (1-4 \delta-r+2 \bar{V}) \int_{0}^{1} f_{1}(\bar{x}) d \bar{x} \\
& \left.+4 \delta \int_{0}^{1} f_{2}(\bar{x}) d \bar{x}\right]+C \int_{0}^{1} f_{3}(\bar{x}) d \bar{x}
\end{aligned}
$$

where $\bar{F}=\left(h_{2}^{2} / \eta_{o} U L B^{2}\right) F$. 
The steady state load capacity $\left(F_{s}\right)$ and centre of pressure $(\overline{\bar{x}})$ can be obtained from the film force and film pressure, respectively, under the steady state (i.e., $h_{m}=h_{m}(0)$ and $V=$ $0)$ as follows:

$$
\begin{gathered}
F_{S}=[F]_{(t=0, V=0)}, \\
\overline{\bar{x}}=\left(\frac{\int_{0}^{B} x p d x}{\int_{0}^{B} p d x}\right)_{(t=0, V=0)} .
\end{gathered}
$$

The dynamic damping coefficient $C_{D}$ and the dynamic stiffness coefficient $S_{D}$ can be obtained as

$$
\begin{aligned}
& C_{D}=\left(\frac{\partial F}{\partial V}\right)_{(t=0, V=0)}, \\
& S_{D}=\left(\frac{\partial F}{\partial h_{m}}\right)_{(t=0, V=0)},
\end{aligned}
$$

which take the dimensionless form:

$$
\begin{aligned}
\bar{C}_{D}= & \frac{h_{2}^{3}}{\eta_{0} L B^{3}} C_{D} \\
= & -12\left(\bar{\eta}+\bar{\eta}_{m}\right)\left[\int_{0}^{1} f_{1}(\bar{x}) d \bar{x}\right. \\
& \left.\quad-\left(\frac{f_{1}(1)}{f_{3}(1)}\right) \int_{0}^{1} f_{3}(\bar{x}) d \bar{x}\right]_{t=0}, \\
\bar{S}_{D}= & \frac{h_{2}^{3}}{\eta_{0} U L B^{3}} S_{D} \\
= & -6\left(\bar{\eta}+\bar{\eta}_{m}\right)\left[(1-4 \delta-r) \int_{0}^{1}\left\{\frac{\partial}{\partial h_{m}} f_{1}(\bar{x})\right\} d \bar{x}\right. \\
& \left.+\left\{C \int_{0}^{1} \frac{\partial}{\partial h_{m}} f_{3}(\bar{x}) d \bar{x}\right\}_{t=0}^{1}\left\{\frac{\partial}{\partial h_{m}} f_{2}(\bar{x})\right\} d \bar{x}\right]_{t=0}+\left\{\frac{\partial C}{\partial h_{m}} \int_{0}^{1} f_{3}(x) d \bar{x}\right\}_{t=0} .
\end{aligned}
$$

\section{Results and Discussion}

To study the ferrohydrodynamic effects on the dynamic characteristics of curved slider bearings, the numerical results for the coefficients of dynamic damping $\left(C_{D}\right)$ and stiffness $\left(S_{D}\right)$ have been calculated. The effect of magnetic field is analyzed by use of parameters $\phi$ and $\alpha$ which stand for the amount of volume concentration of particles and Langevin parameter, respectively. In the analysis, $\alpha=0$ and $\phi=0$ refer to the cases of "no magnetic field" and common lubricants (nonmagnetic lubricant), respectively. In order to validate the present results and to enhance its practical applicability, the steady state load carrying capacity of simple inclined slider $(\delta=0)$ is compared with the results of Taylor and Dowson [20] in Figure 2.

The values of the parameters taken in the present analysis are as follows:

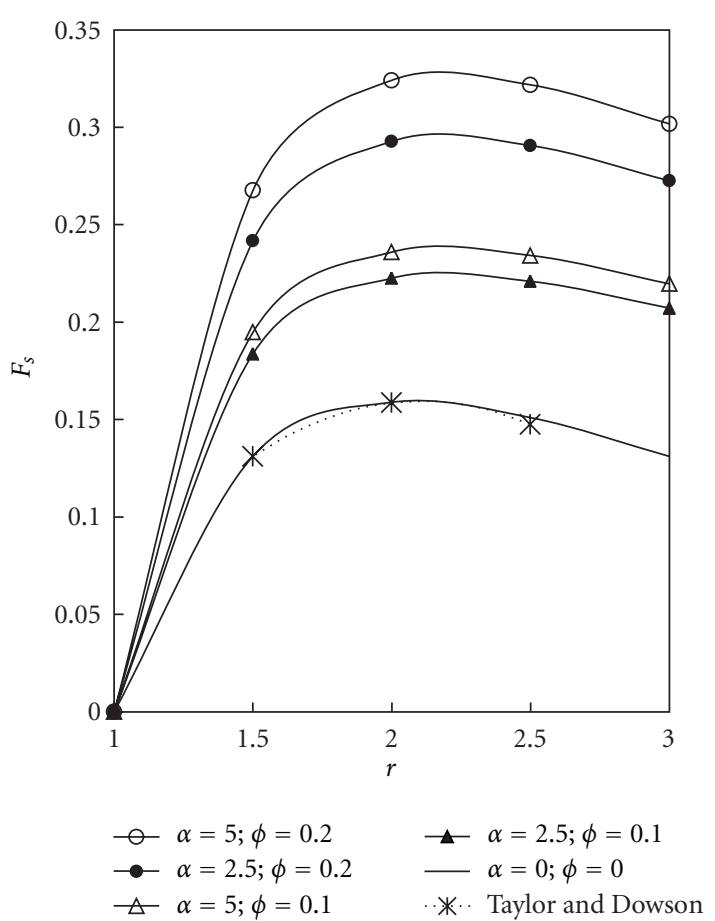

FIGURE 2: Variation of steady-state load capacity of plane inclined slider with respect to film thickness ratio $r$ for different values of $\alpha$ and $\phi$.

(i) $\alpha: 0-10$, (ii) $\phi: 0-1.0$, (iii) $\delta: 0-0.5$, and (iv) $r: 1.0-2.0$,

Figure 2 shows the variation of dimensionless steady state load capacity with respect to the inlet outlet film thickness ratio $r$ for plane inclined slider $(\delta=0)$ compared with the results of Taylor and Dowson [20]. It is clear from the figure that the load carrying capacity obtained in the present analysis for ferrohydrodynamic parameters $\alpha=0, \phi=$ 0 is in the good agreement with the results obtained by Taylor and Dowson [20]. Further, it is observed that the load capacity increases with the increase of Langevin parameter $\alpha$ as well as the volume concentration parameter $\phi$.

Figure 3 presents the variation of dimensionless dynamic damping coefficient $\bar{C}_{D}$ with respect to the volume concentration parameter $\phi$ for Langevin parameter $\alpha=$ $0,2.5,5,7.5,10$ and curvature parameter $\delta=0,0.5$. It is observed that the coefficient of dynamic damping $\left(\bar{C}_{D}\right)$ increases with the increase of $\phi$ in both the cases: (i) when the magnetic field is absent $(\alpha=0)$ and (ii) when the uniform transverse magnetic field is applied $(\alpha>0)$. However, the presence of magnetic field increases the damping coefficient which further increases with the increase of intensity of magnetic field $(\alpha)$, that is, the value of damping coefficient is higher for higher values of Langevin parameter $\alpha$. Further, the nature of variation of damping coefficient for plain and curved sliders $(\delta=0$ and $\delta=0.5)$ is found similar.

Figure 4 presents the variation of dimensionless dynamic stiffness coefficient $\bar{S}_{D}$ with respect to the Langevin parameter $\alpha$ for $\phi=0,0.1,0.2,0.3,0.4$ and curvature parameter $\delta=$ $0,0.5$. It is observed that the coefficient of stiffness obtained 


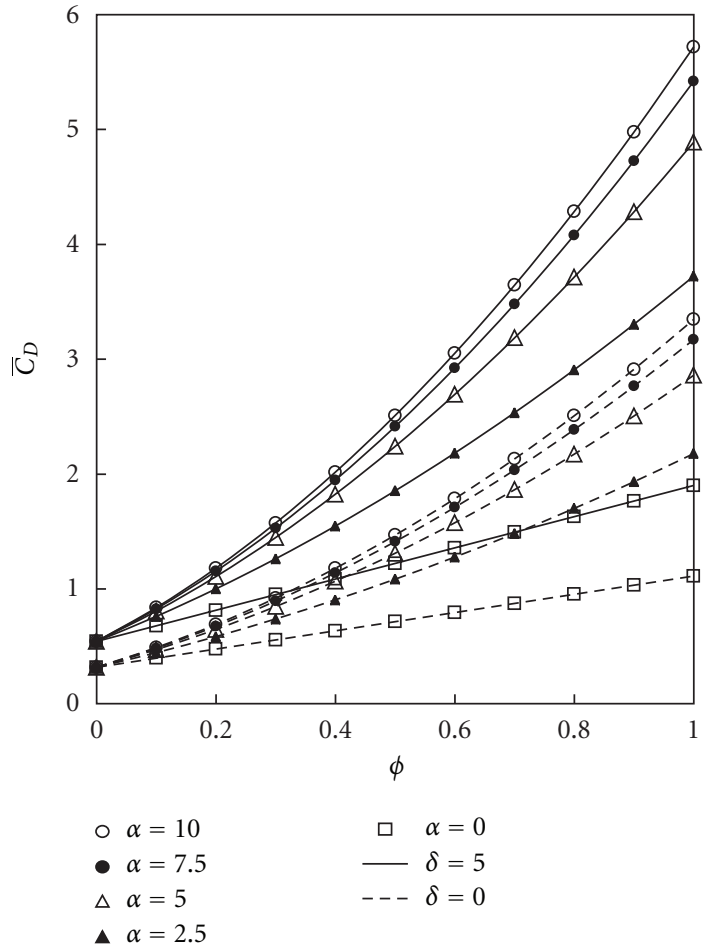

Figure 3: Variation of dynamic damping coefficient with respect to volume concentration parameter $\phi$ for film thickness ratio $r=2$ and different values of $\alpha$.

with common lubricants $(\phi=0)$ is less than that of a ferrofluid based lubricant regardless of the intensity of the magnetic field $(\alpha)$. Further, with the ferrofluid analysis, the stiffness increases with the increase in Langevin parameter $(\alpha)$. It is also observed that the stiffness increases with $\alpha$ at a higher rate upto $\alpha \approx 2$ and for $\alpha>4$, the effect of increasing the field intensity (i.e., increasing $\alpha$ ) decays in a sequential manner. The results are still sustained for both the plain and curved sliders $(\delta=0$ and $\delta=0.5)$.

\section{Conclusions}

In the present theoretical investigation, the dynamic characteristics of curved slider bearings are studied in the presence of transverse magnetic field. The ferrofluid model given by Shliomis is adopted to obtain modified Reynolds equation. Based on the results, so obtained, the following conclusions have been drawn.

(1) Magnetic fluid under the transverse magnetic field provides an improvement in the dynamic stiffness and damping characteristics of slider bearings.

(2) On comparing with the conventional lubricants, both the dynamic stiffness and the dynamic damping coefficients are higher for ferrofluids even if the magnetic field is absent.

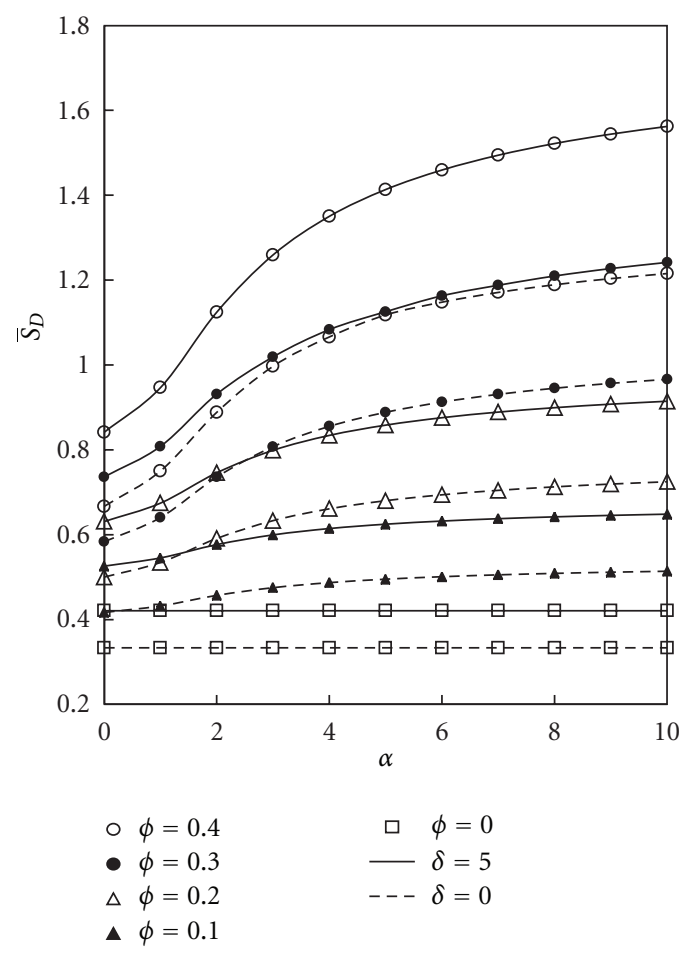

FIGURE 4: Variation of dynamic stiffness coefficient with respect to Langevin parameter $\alpha$ for film thickness ratio $r=2$ and different values of $\phi$.

(3) Under the applied magnetic field, both the stiffness and the damping increase with the increase of volume concentration of magnetic particles.

\section{Nomenclature}

$B$ : $\quad$ Length of the bearing

$C_{D}, \bar{C}_{D}$ : Dynamic damping coefficients,

$\bar{C}_{D}=\left(h_{2}^{3} / \eta_{o} L B^{3}\right) C_{D}$

$F, \bar{F}: \quad$ Film force, $\bar{F}=\left(h_{2}^{2} / \eta_{o} U B^{2} L\right) F$

$\bar{F}_{s}: \quad$ Dimensionless steady state load capacity

$h, \bar{h}: \quad$ Film thickness, $\bar{h}=h / h_{2}$

$h_{m}, \bar{h}_{m}$ : Time dependent minimum film thickness, $\bar{h}_{m}=h_{m} / h_{2}$

$h_{s}, \bar{h}_{s}: \quad$ Steady state film thickness, $\bar{h}_{s}=h_{s} / h_{2}$

$\vec{H}: \quad$ Applied magnetic field

I: $\quad$ Sum of moments of inertia of the particles per unit volume

$k_{B}$ : Boltzmann constant

$L: \quad$ Width of the bearing

$m$ : Magnetic moment of a particle

$\vec{M}: \quad$ Magnetization vector

$n$ : $\quad$ Number of magnetic particles per unit volume

$p, \bar{p}: \quad$ Film pressure, $\bar{p}=p h_{2}^{2} /\left(\eta_{o} U B\right)$

$r: \quad h_{1} / h_{2}$,

$\vec{S}: \quad$ Internal angular moment 
$S_{D}, \bar{S}_{D}:$ Dynamic stiffness coefficients,

$\begin{array}{ll} & \bar{S}_{D}=\left(h_{2}^{3} / \eta_{o} U L B^{3}\right) S_{D} \\ t, \bar{t}: & \text { Time, } \bar{t}=U t / B \\ T: & \text { Temperature } \\ \vec{v}: & \text { Velocity vector } \\ \bar{V}: & d h_{m} / d t \\ x, \bar{x}: & \text { Coordinate distance, } \bar{x}=x / B \\ \overline{\bar{x}}: & \text { Centre of pressure } \\ \alpha: & \text { Langevin parameter } \\ \delta: & H_{c} / h_{2}\end{array}$

$\eta, \bar{\eta}: \quad$ Effective viscosity (Shliomis model), $\bar{\eta}=\eta / \eta_{0}$

$\eta_{m}, \bar{\eta}_{m}:$ Additional viscosity due magnetization, $\bar{\eta}_{m}=\eta_{m} / \eta_{o}$

$\eta_{o}: \quad$ Viscosity of the carrier fluid

$\mu_{o}: \quad$ Free space permeability

$\vec{\Omega}: \quad$ Local angular velocity

$\phi: \quad$ Volume fraction of the dispersed solid phase

$\rho: \quad$ Fluid density

$\tau_{s}: \quad$ Spin relaxation time

$\tau_{B}: \quad$ Brownian relaxation time of magnetic particles.

\section{Acknowledgment}

The authors, hereby, thank Dr. V. K. Kapur (Former Professor and Chairman, K. N. Institute of Technology, Sultanpur, India) for providing useful materials and the valuable guideline to enhance the content of this paper.

\section{References}

[1] R. S. Gupta and P. Kavita, "Analysis of rotation in the lubrication of a porous slider bearing: small rotation," Wear, vol. 111, no. 3, pp. 245-258, 1986.

[2] U. P. Singh and R. S. Gupta, "On the performance of pivoted curved slider bearings: rabinowitsch fluid model," in Proceedings of the National Tribology Conference (NTC'11), p. 24, IIT Roorkee, 2011.

[3] M. D. Pascovici, A. Predescu, T. Cicone, and C. S. Popescu, "Experimental evidence of cavitational effects in a Rayleigh step slider," Proceedings of the Institution of Mechanical Engineers, Part J, vol. 225, no. 6, pp. 527-537, 2011.

[4] K. Venkateswarlu and C. M. Rodkiewicz, "Thrust bearing characteristics when the slider is approaching terminal speed," Wear, vol. 67, no. 3, pp. 341-350, 1981.

[5] P. D. Williams and G. R. Symmons, "Analysis of hydrodynamic slider thrust bearings lubricated with non-newtonian fluids," Wear, vol. 117, no. 1, pp. 91-102, 1987.

[6] R. K. Sharma and R. K. Pandey, "Experimental studies of pressure distributions in finite slider bearing with single continuous surface profiles on the pads," Tribology International, vol. 42, no. 7, pp. 1040-1045, 2009.

[7] V. K. Kapur, "Magneto-hydrodynamic pivoted slider bearing with a convex pad surface," Japanese Journal of Applied Physics, vol. 8, no. 7, pp. 827-835, 1969.

[8] M. E. Shimpi and G. M. Deheri, "A study on the performance of a magnetic fluid based squeeze film in curved porous rotating rough annular plates and deformation effect," Tribology International, vol. 47, pp. 90-99, 2012.
[9] N. C. Das, "A study of optimum load-bearing capacity for slider bearings lubricated with couple stress fluids in magnetic field," Tribology International, vol. 31, no. 7, pp. 393-400, 1998.

[10] R. B. Kudenatti, D. P. Basti, and N. M. Bujurke, "Numerical solution of the MHD reynolds equation for squeeze film lubrication between two parallel surfaces," Applied Mathematics and Computation, vol. 218, pp. 9372-9382, 2012.

[11] K. Raj, B. Moskowitz, and R. Casciari, "Advances in ferrofluid technology," Journal of Magnetism and Magnetic Materials, vol. 149, no. 1-2, pp. 174-180, 1995.

[12] J. T. Jenkins, "A theory of magnetic fluids," Archive for Rational Mechanics and Analysis, vol. 46, no. 1, pp. 42-60, 1972.

[13] M. I. Shliomis, "Effective viscosity of magnetic suspensions," Soviet Physics, vol. 34, pp. 1291-1294, 1972.

[14] M. I. Shliomis, "Magnetic fluids," Soviet Physics, vol. 17, pp. 153-169, 1974.

[15] P. Ram and P. D. S. Verma, "Ferrofluid lubrication in porous inclined slider bearing," Indian Journal of Pure and Applied Mathematics, vol. 30, no. 12, pp. 1273-1281, 1999.

[16] R. C. Shah and M. V. Bhat, "Ferrofluid squeeze film between curved annular plates including rotation of magnetic particles," Journal of Engineering Mathematics, vol. 51, no. 4, pp. 317-324, 2005.

[17] H. Yamaguchi, Engineering Fluid Mechanics, Springer, Amsterdam, The Netherlands, 2008.

[18] J. R. Lin, "Derivation of ferrofluid lubrication equation of cylindrical squeeze films with convective fluid inertia forces and application to circular disks," Tribology International, vol. 49, pp. 110-115, 2012.

[19] S. Abramovitz, "Theory for a slider bearing with a convex pad surface; side flow neglected," Journal of the Franklin Institute, vol. 259, no. 3, pp. 221-233, 1955.

[20] C. M. Taylor and D. Dowson, "Turbulent lubrication theoryapplication to design," Journal of Lubrication Technology, vol. 96, no. 1, pp. 36-47, 1974. 

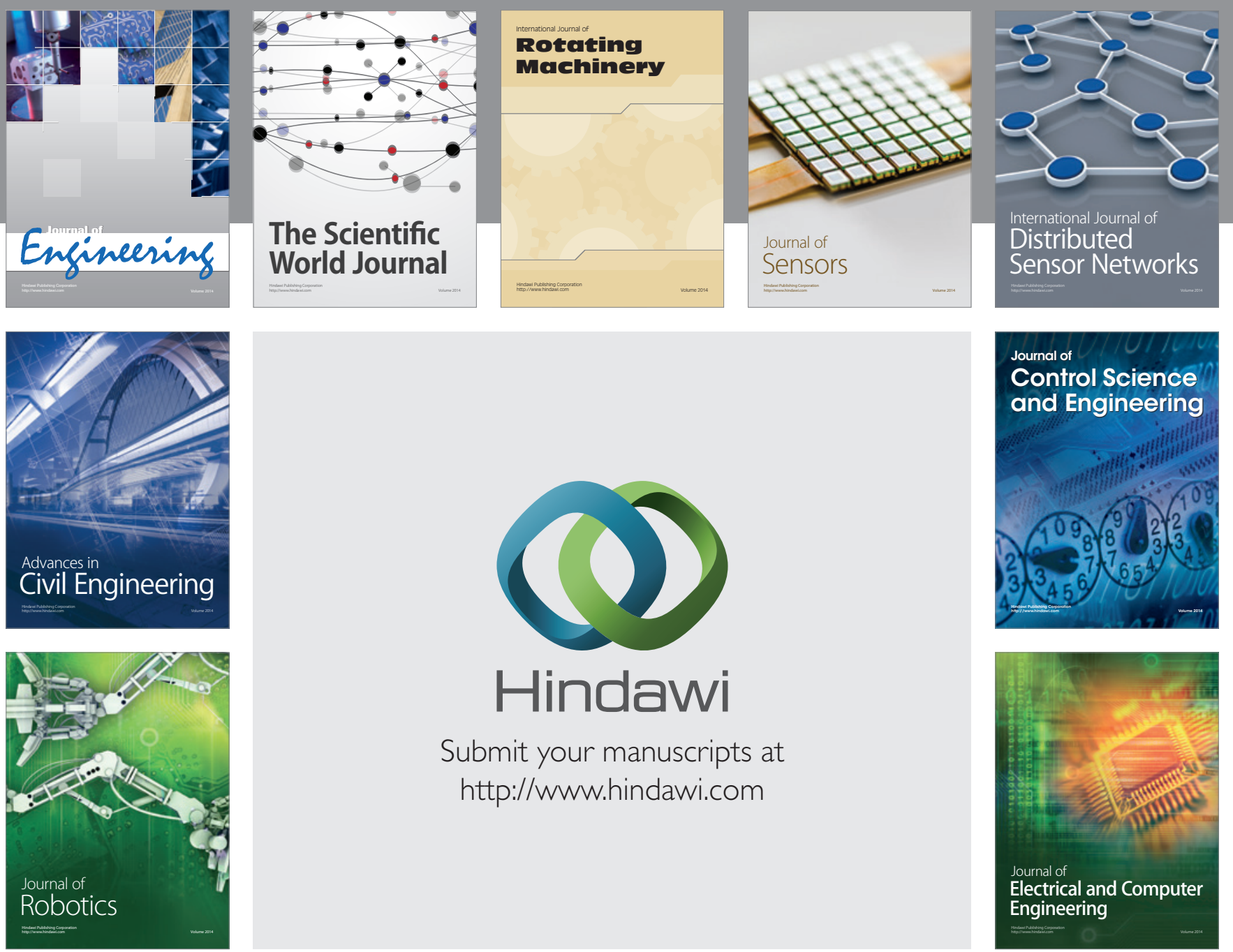

Submit your manuscripts at

http://www.hindawi.com
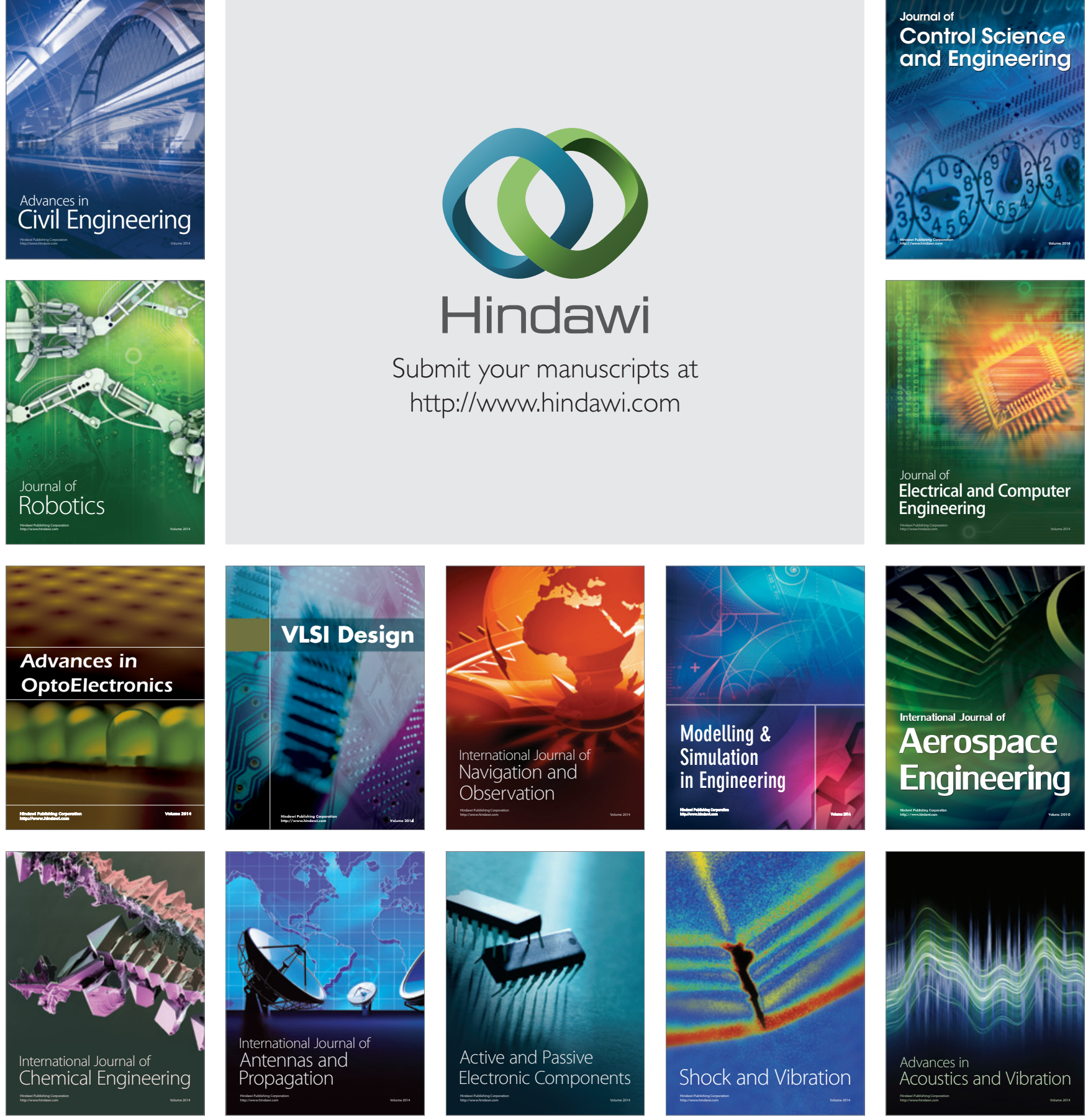